\title{
Formally Modeling Pretend Object Play
}

\author{
Alexander Zook \\ School of Interactive \\ Computing \\ Georgia Institute of \\ Technology \\ a.zook@gatech.edu
}

\author{
Brian Magerko \\ School of Literature, \\ Communication, and Culture \\ Georgia Institute of Technology \\ magerko@gatech.edu
}

\author{
Mark Riedl \\ School of Interactive \\ Computing \\ Georgia Institute of \\ Technology \\ riedl@gatech.edu
}

\begin{abstract}
We address the problem of building computational agents that are capable of play. Existing research has examined the forms, characteristics, and processes involved in various kinds of play at a high level. However, this research does not provide a unified framework at a level of detail sufficient for building computational agents that can play. As a step toward addressing this gap we synthesize diverse research on pretend play to recognize important components of pretend play agents. We also develop a formal model of one key component of pretend play, pretend object play, and present a computational implementation of this model. Our work provides initial criteria for the content and processes necessary for pretend play agents.
\end{abstract}

\section{Author Keywords}

play, games, creativity, artificial intelligence

\section{ACM Classification Keywords}

I.2.0 [Artificial Intelligence] General---Cognitive simulation; I.2.1 [Artificial Intelligence] Applications and Expert Systems---Games

\section{General Terms}

Design, Algorithms

\section{INTRODUCTION}

What knowledge and processes are necessary for building an agent that can play? Existing play research has examined diverse aspects of play, including the forms, characteristics, and processes involved in a variety of kinds of play. Pretend play is an exemplar of everyday creativity - the generation of creative output by normal individuals in mundane contexts [3]. Yet play research to date falls short of providing a unified model of play at a level of detail sufficient for building computational agents. Understanding play at the computational level can drive more detailed explanations of play, connect play to other domains of human activity, particularly creativity, and enable technologies that leverage these detailed models in

Permission to make digital or hard copies of all or part of this work for personal or classroom use is granted without fee provided that copies are not made or distributed for profit or commercial advantage and that copies bear this notice and the full citation on the first page. To copy otherwise, or republish, to post on servers or to redistribute to lists, requires prior specific permission and/or a fee.

C\&C'11, November 3-6, 2011, Atlanta, Georgia, USA.

Copyright 2011 ACM 978-1-4503-0820-5/11/11...\$10.00. supporting human play and creativity.

Creativity research has typically focused on the generation of novel or innovative artifacts such as music, paintings, architectural designs, or engineered artifacts by highperforming individuals. Yet both children and adults routinely engage in related creative processes when playing. During play activities individuals incorporate knowledge from multiple domains to co-construct interesting experiences or products [7,16,25,32,36]. Players devise and enact amusing stories, build kingdoms in sand, and conjure imaginary playmates.

Play and playful activities are also known to contribute to important everyday skills: emotional expression and regulation, self-exploration and social comparison, group dynamics and problem solving, training for social roles, and divergent thinking [27]. Unstructured activities and self-structured activities in particular have been found to benefit cognition and education [18,23,28]. Play appears to involve fundamental processes that contribute to a variety of human behaviors.

Playful behavior is closely connected to creative activity. Play involves many features of central importance to creative cognition and interaction, including: evolving and highly contextual processes, interactions and negotiations among multiple individuals, open-ended frames for interaction, and the interaction among real and pretend frames. The process of play requires highly contextualized cognitive processes responding to a constantly evolving frame of activity, rather than working within a predefined and fixed space [31]. During play multiple individuals are continually required to negotiate the terms of the activity with one another, rather than working solely within their own mental framework. These negotiations result in openended frames for the play activity that are constantly cocreated. A central aspect of play interactions and the evolving play activity is the interplay between the realworld space and the pretend domain being used. Interacting individuals must continually communicate and reason about both levels of an activity, rather than escaping to a purely conceptual domain.

Despite the known benefits of play the number of spaces and opportunities for free, unstructured play has diminished. The extent to which children are allowed to freely explore in urban areas without supervision has 
consistently decreased over the last several decades [15]. In response several movements have attempted to mitigate these limitations or leverage the benefits of play, including play therapy for adults and children, construction of playgrounds, and the incorporation of more self-directed and less structured forms of activities into educational systems [23]. All attempts to leverage play to enhance flexibility and the ability to effectively handle novel experiences have been based on high-level frameworks of play activity.

Understanding play is particularly important since artificially intelligent agents are increasingly part of the everyday world of human interactions. Researchers in social robotics have begun to explore how robots can interact with humans and other robots in socially appropriate and meaningful ways [1]. Examples range from the emotional modeling and affective communication explored by Breazeal's Kismet robot [4,5,6] to the RoboticsLab's development of more autonomous and adaptive robots with the Maggie robot [34]. Play research can complement these efforts by examining the key cognitive and behavioral processes involved in free-form interaction and make-believe. Understanding play computationally can enable the development of agents that playfully interact both with humans and other agents, complementing existing efforts to increase access to playful interactions.

Play research to date has established the high-level theoretical and conceptual models of play mentioned above. The play scholar Sutton-Smith, in his comprehensive review of the play literature, has argued the next step to understanding play will be the development of detailed processual accounts of play [36]. Processual accounts must push into providing detailed models of the cognitive, social, and affective processes involved in play. Without such models we cannot understand how play relates to such a diversity of human activities. Further, computational agents that engage in play require exactly this level of detail in process and content to be realized.

We propose the development of computational play to formally understand the processes and content of play through the lens of computation. Computational play focuses on creating detailed models of play activities that are grounded in theoretical and empirical research on play and human cognition. Developing such models can address the knowledge and processes used in play, benefiting play research and enabling the creation of playful agents. Play research can be extended to understand the underlying aspects that unify diverse forms of play and link play to other domains of human activity. Computational agents can be used to leverage the many benefits of play.

We present two primary contributions in this work. First, we unify diverse research on play and cognition in the category of pretend play to identify its key representations and processes. Second, we develop a formal model of pretend object play, a necessary component of pretend play, as a first step toward formally grounding this framework.

We develop these contributions as follows. We first synthesize play research on ontologies, definitions, and core processes of play to develop the key content and processes involved in pretend play. We argue that pretend play provides a domain to examine situated and dynamic creative cognition, connecting play to the processes of analogy and conceptual blending. In order to ground these high-level models we next focus on a key component of pretend play, the pretend use of objects. We develop a formal model of pretend object play and present a computational implementation of this model. We conclude by outlining additional developments needed to develop a formal model of pretend play.

\section{SYNTHESIZING PRETEND PLAY}

In this section we synthesize play research toward a unifying framework for pretend play agents. Pretend play is characterized by dual representations of the real and pretend domains of interest. Pretend domains contain knowledge about particular themes, scripts, and rules for activities. The pretend and real domains are connected and blended together in play, with these combinations balancing constraints of coherence against free-form change. Communicating about both pretend and real domains simultaneously requires the process of metacommunication.

\section{Play Ontologies}

Many researchers have examined the various kinds of play activities and organized these by their primary characteristics. Sutton-Smith organized play activities into several categories based on the existing play literature [36]:

- mind or subjective play (e.g. imagination)

- $\quad$ solitary play (e.g. collecting objects)

- playful behaviors (e.g. pranks)

- informal social play (e.g. joking, parties)

- $\quad$ vicarious audience play (e.g. spectator sports)

- $\quad$ performance play (e.g. performing music)

- celebrations and festivals (e.g. Mardi Gras)

- $\quad$ contests (games and sports) (e.g. athletics, casinos)

- $\quad$ risky/deep play (e.g. caving)

Brown and Vaughan [7] recognized nine major categories of play derived from their research:

- attunement - mother-infant connection formation

- body play and movement - moving in the world

- $\quad$ object play - manipulating objects

- $\quad$ rough and tumble play - e.g. playful wrestling

- belonging play - developing social connections

- celebratory play 
- $\quad$ pretend play - creating one's own sense of the world

- $\quad$ storytelling play - conveying a sense of the world to others

- creative play - realizing an envisioned change in oneself or the world

Among these we focus on pretend play as an exemplar of the creative processes involved in play. Pretend play corresponds roughly to Sutton-Smith's mind play and Brown and Vaughan's pretend play.

Pretend play - also known as fantasy play, sociodramatic play, or symbolic play - is play that involves constructing and enacting scripts and roles that children draw from life experiences and media sources such as fairy tales, books, and television [26]. Children socially interact and communicate with peers during the dramatic enactment and co-construction. Pretend play involves many of the commonly recognized instances of creativity in play, ranging from imaginary friends to playful re-enactments and alterations of popular media stories. While understanding pretend play will not unify all forms of play, the emphasis on interaction makes this domain particularly conducive to the development of interactive play agents.

\section{Play Definitions}

Definitions of play recognize play as involving both pretend and real domains. These definitions have emphasized an important duality between structure and freedom in play. As such, pretend play agents will need models of these two domains and the process of balancing freedom and constraint involved in pretend play.

The cultural historian Huizinga provided one of the earliest definitions of play, emphasizing its social manifestations [17]. He defined play as: outside normal life; non-serious, but absorbing; not involving material interest; bounded in time and space; including fixed rules and an orderly manner; promoting formation of social groupings surrounded with secrecy; and having groups that stress their difference from the normal world. These criteria were based on his focus on play as underlying the formation of human culture.

The sociologist Caillois [8] agreed with Huizinga's emphasis on the social aspects of play, but disagreed with his narrow focus on mystery and ritual. Caillois sought to ground a definition of play in understanding the difference between acting a role and enacting a situation. This distinction captures the difference of the as if attitude of theatre, where roles are played, and the for real attitude of sports, where games are enacted. Caillois found play has six primary traits, being:

- free - not obligatory

- $\quad$ separate - limited in time and space

- uncertain - leaving uncertainty in outcomes

- $\quad$ unproductive - not yielding goods
- governed by rules - having special regulations

- make-believe - requiring awareness of a second reality

Using this definition of play, Caillois expounded on a model of games using two dimensions: the rubric of the game and the attitude taken towards the game. Game rubrics consisted of the categories of competition (agôn), chance (alea), pretend (mimicry) and vertigo (ilinx). Games of competition and chance both seek equality among players, with the former differentiating players by skill and the latter by luck. Pretend games seek to convince an audience of something. Vertigo games are based around the pleasure of disorientation, such as in roller-coaster rides. Each of these rubrics can be approached either with a rulebased (ludus) or improvisational (paidia) attitude. Ludus emphasizes the concrete game being played, strict adherence to rules, and explicit structure. Paidia instead focuses on personal experience, the more abstract fantasy realm being used, and spontaneous change. Caillois sought to organize play by both the structure of the activity and the particular attitude taken towards it.

Fromberg's [13] review of early childhood play research found children's play is:

- $\quad$ symbolic - representing reality in "as-if" or "whatif" terms

- meaningful - in connecting or relating experiences

- active - in children doing things, including imagining

- pleasurable - even if children are seriously engaged in the activity

- voluntary

- intrinsically motivated - regardless of drive by curiosity, mastery, affiliation, or others

- rule-governed - whether implicitly or explicitly expressed

- $\quad$ episodic - in having emerging and shifting goals that children develop spontaneously

As in Huizinga and Caillois's definitions, Fromberg recognizes the importance of structure (rule-governed) and freedom (symbolic, voluntary, intrinsically motivated).

A common thread among play, creativity, and cognition research is the nature-nurture or innate-learned distinction. In psychology, this relates to the extent to which a given behavior or trait can be explained by fixed innate aspects or changing and developed features. Play definitions fall along a similar spectrum ranging from fixed and predetermined (nature, innate) to flexible and acquired (nurture, learned). In play this spectrum manifests as extending from fixed games to open-ended play or improvisation.

Structured perspectives on play emphasize the scripted and rule-based nature of play. Huizinga, Caillois, and Fromberg 
all see play as involving orderly processes or episodes and also recognize rules that govern play activities. Caillois's ludus and games of competition and chance emphasize highly structured forms of play based strictly in conventionalized rules. These structures parallel Boden's emphasis on an accepted set of generative structures that bound a creative domain [3]. Pretend play agents will need the ability to recognize and enforce rule structures based on a given activity. These must include both explicitly declared game rules as well as implicit conventions of play including social and cultural context.

Play also involves open-ended, improvisational aspects. Huizinga, Caillois, and Fromberg all recognize play as free or intrinsically motivated. Freedom in these accounts is based on play involving a separate domain or frame outside the context of normal activity. Caillois's paidia and games of pretend and vertigo all emphasize play as not fully tethered to external constraints and circumstances, working within a subjective, make-believe realm or loosening connections to the physical realm. Both Caillois and Fromberg emphasize play as symbolic. Play is free and creative by working with an additional mental domain that is not simply the physical space of activity. Activities are fluid in using make-believe to manipulate an ongoing activity, drawing from other domains of knowledge. Fauconnier and Turner's conceptual blending describes the similar process of combining domains for creative results $[10,11]$. Thus, pretend play requires the capacity for modeling a separate make-believe domain that maintains some connections to the real domain and some connections to other make-believe content.

\section{Metacommunication, Analogy and Blending}

While the above definitions recognize the dual domains of pretend and real in play, they do not give detailed accounts of the behavioral or cognitive processes involved in relating these domains and communicating about these relations to other players. Pretend play agents require models of the specific content of make-believe domains and the processes of connecting, combining, and communicating about these domains.

\section{Pretend Domain Content}

Empirical research on children and adults at play provides the types of content that comprise the make-believe domain. Both Sawyer [32] and Horst et al. [16] have found that children at play engage in a process of appropriating elements of their surrounding media culture for development of their play activities. Children borrow major themes, roles and narratives from television, fairy tales and other sources. Real-life activities are also incorporated into play as loose scripts. In addition, rules based on analog and digital games are used to structure play activities. SuttonSmith found that $90 \%$ of children's descriptions of play with toys focused on pretending, personal narratives and dialogues in play, rather than the specific characteristics of the toys themselves [35]. Children thus selectively borrow and modify content from their everyday life and media environment to suit their ends, and draw from many sources when constructing any given activity. In the context of creativity research this work provides examples of the processes involved in combining, exploring and modifying conceptual domains [3]. Pretend play agents thus need models of outside domains of content to inform their make-believe frames. These outside domains should provide knowledge of themes, event scripts, and rule structures.

\section{Metacommunication}

Players constantly draw from outside domains to add structure and content to their ongoing activity, communicating these changes to one another. The central process of navigating between the pretend and real aspects of play is metacommunication. The cyberneticist and anthropologist Bateson [2] found play activities constantly involve communication at two levels: the real-world activity and the pretend domain being used. Metacommunication is the process of communicating at both of these levels simultaneously to regulate interaction. This involves both communicating about a real-world action and communicating about that communication to connect it to a pretend domain. When a dog nips another dog on the ear during play it communicates the real-world action of nipping and metacommunicates the pretend action of biting.

Sawyer [31] notes that play actions are more properly understood as being metapragmatic. In linguistics, communication uses language to denote aspects of the world, referring to the direct meaning of a word or expression. Pragmatics involves use of language at the level of framing or contextualizing communication. Communication involves relatively fixed and shared meanings, while pragmatics emphasizes fluid and contextual meanings. Metacommunication refers to content of an activity, while metapragmatics refers to the frame of an activity or conversation. Metapragmatics and metacommunication are the means by which agents can arrive at a shared understanding of a given situation, here a pretend scenario [14]. Metacommunication underlies the processes of understanding given objects within a play session, while metapragmatics investigates the ongoing changes made to the framing of that session. Sutton-Smith describes this distinction as the difference between play manipulating the content of a given activity - and metaplay - manipulating the structure of an activity itself [36]. Playful creativity can involve both of these domains and pretend play agents will need the ability to manipulate both the content and frame of a play activity.

Sawyer [31] has provided an empirically grounded formulation of the pretend play process as improvisation. He grounds this formulation in two key concepts: indexical presuppositions and indexical entailments. Indexes are signs that require an association between the sign and its object. Sawyer examines speech acts in play that are indexical in referring to aspects of the play context, such as 
recognizing a shared topic. For example, a play interaction can have the context, or frame, of playing house. Indexical presuppositions refer to accepted frame aspects of an ongoing interaction. A child may draw on a presupposed setting or role, such as a country home or being a mother. Indexical entailments are statements that convey a potential direction for an interaction. Children may propose new relations among their characters such as one player being a mother with another player as her baby. Over the course of a play session all players constantly balance between the given structure of indexical presuppositions and changes to that structure possible with indexical entailments. Thus, a child enacting a mother must balance between enacting the motherly role while proposing new activities to play out, such as feeding her baby or cooking.

Indexical presuppositions and entailments can serve as the elements agents use when communicating to one another during a play activity regarding the content and frame of the activity. These do not specify, however, the process of interrelating the pretend and real domains. When an agent pretends that a cardboard tube is a sword it must simultaneously reason about properties of the real cardboard tube, pretend sword, and the relation between them within the context of the play activity. This reasoning must be used to drive actions that in turn convey information regarding all these same aspects. A model of pretend play requires an account of how an individual maps between the real and pretend frames and reasons about their relationship.

\section{Analogy}

Recognizing and making analogies involves comparing the conceptual structure of two domains. Analogical reasoning can enable pretend play agents to find connections between a real and pretend domain. In making or recognizing an analogy an agent must build a mapping between the conceptual structures of two domains in order to interpret one domain in terms of the other. Two computational models of analogy are SME [9] and MAC/FAC [12]. SME is based on recognizing matched systems of relations among concepts, emphasizing shared structure over particular features. It models the process of building a mapping between two given concepts. For example, SME maps from the solar system to an atom by recognizing their similar processes of revolving objects and forces of attraction. The mapping pairs the sun to the nucleus of the atom and planets to the electrons of the atom. MAC/FAC extends the SME model by incorporating a model of retrieval of potential analogies using a simplified, nonstructural filtering process, before using a full structuremapping process to evaluate the quality of resulting analogies.

\section{Conceptual Blending}

Conceptual blending accounts for the unconscious process of combining features and relations of concepts to yield novel concepts [11]. Pretend play leverages conceptual blending when reasoning about objects that mix features of the real and pretend contexts of an activity. Conceptual blending theory starts from given conceptual spaces consisting of features and structures and explains the constraints and processes involved in combining these spaces to yield novel results. Specifically, two (or more) input conceptual spaces are linked via a third generic space used to map among these spaces. Components of these spaces are selectively mapped into a resulting fourth blended space. Reasoning within the blend space can be propagated back to the input spaces.

As an example, a modern boat, Great America II, sailing from San Francisco to Boston in 1993 was compared to a historical one, Northern Light, which made the same trip in 1853 [10]. Observers at one point noted: "At this point, Great America II is 4.5 days ahead of Northern Light." To make this comparison a blend with both boats occupying a shared space starting at the same time is constructed. Based on this blend, inferences about the relative positions of the boats can be made and mapped back to the input space of the Great America II's current trip to infer its lead in time.

Veale's SAPPER [37] is based on a bridge-building model of structure mapping that recognizes local regularities of structure and bridges these elements. The most elaborate local model of similarity serves as an anchor for mapping between the domains, with additional consistent mappings being folded into this structure. The final structure represents a blended result. SAPPER's process of matching, selecting a best match, and extending that match by adding in additional relevant features can ground the process of blending the real and pretend domains for the purpose of pretend play. The Riu system has used a similar process to model imaginative remembering using memories structured using force-dynamic models, supporting the viability of this approach for navigating imagined and real domains [38].

\section{PRETEND OBJECT PLAY}

The research above synthesizes key aspects of pretend play that hold across many concrete domains needed for pretend play agents. We have drawn from several studies of pretend play with objects to formulate an account of this particular pretend play activity at a sufficient level of detail for building a computational model.

\section{Pretend Object Play Research}

Pretend object play is the process of using real-world objects as substitutes for pretend objects as part of a pretend scenario, a core component of pretend play activities [25]. Modeling pretend object play requires specifying how objects are represented when making substitutions and the substitution process of relating and reasoning about the pretend object referenced and the real object used.

\section{Object Representation}

Sawyers and Carrick [33] note that four levels of object substitution occur in children's pretend play: functional, high-level prototypical, low-level prototypical, and 
insubstantial. Functional substitution is the use of an object in its intended or real-world manner. An example is using a real pen as a pretend pen. High-level prototypical substitution is the use of a real-world object that closely resembles the desired pretend object, such as using a replica sword as a pretend sword. Low-level prototypical substitution involves referring to a pretend object with a real object that does not closely resemble it, such as using a cardboard tube for a pretend sword. Insubstantial substitution involves communicating through words or gesture about pretend objects without real object proxies. An example is miming the presence of a sword by shaping one's hands as if holding a handle and acting out swordswinging motions.

Both Sutton-Smith's research on toys and Sawyers and Carrick's research on pretend players found low-level prototypical substitutions to be the most common in play activities, aligning with Montessori schooling techniques that find such objects most conducive to creative play $[23,33,35]$. Our model focuses on low-level prototypical substitutions in our agent model. High-level prototypical and functional substitutions both de-emphasize the processes involved in reasoning about the relations of the pretend and real domains by narrowing any distinction between these realms. Insubstantial substitutions instead emphasize the properties of gestures and verbal communication, losing the grounding role of particular objects as props. By modeling low-level prototypical object substitutions we intend to explore how agents work within the joint real and pretend domains.

We represent objects by combining insights from Rosch's [29] work on cognitive models of categorization and Lakoff and Johnson's [20] work on mental representations used in metaphors. Rosch found humans employ a graded model of categorization of objects, seeing members of categories as more or less central to a group. Drawing from this insight, we represent objects using a set of fuzzy attributes. Fuzzy set theory relaxes the assumption of binary membership values used by classical set theory, instead allowing realvalued degree-of-membership values [19]. Intuitively this captures the common-sense notion that cognitive models of objects classify them as being more or less members of many categories, rather than being exclusively limited to disjoint sets [21]. Fuzzy modeling techniques have been used to support agents capable of interacting with humans in improvisational domains [24] as well as for machine reasoning about numerical data [22]. As an example, a sword has a 0.8 (on a [0-1] scale) degree-of-membership (DOM) value for stabbing, as swords are prototypical objects used for stabbing. Swords have only a 0.3 DOM value for size, reflecting their relative smallness compared to humans. Note that all real dimensions are scaled to have 0.5 DOM values as representing "human-scale" dimensions.

The set of attributes we have chosen is derived from Lakoff and Johnson's work on the properties of objects used to drive metaphorical understanding [20]. Lakoff and Johnson argue that humans understand objects in terms of four sets of interactional properties. Interactional properties are sets of properties of objects derived from interacting with these objects, rather than abstract concepts not grounded in the real world. Lakoff and Johnson term such sets of properties dimensions, in recognition of their shared features. The four dimensions of interactional properties are: (1) perceptual, (2) functional, (3) motor-activity, and (4) purposive properties. Perceptual properties capture perceived features of objects. Perceptual properties include the size of an object, its color, and shape. Functional properties capture uses of objects as mechanisms or means towards ends. For example, chairs function to allow sitting while guns perform shooting. Motor-activity properties capture ways in which the object can be readily manipulated. Motoractivity properties include ease of gripping an object, object flexibility, and object brittleness. Purposive properties involve the ends towards which objects are applied. For example, a blanket may be used to keep warm, conceal an object, or to bundle objects together for carrying.

Based on Sawyers and Carrick's findings that children typically rely on resemblance of physical properties of objects when using them for low-prototypicality substitution we do not represent functional properties of the real objects being used. This captures the notion that most low-prototypicality objects are used for their appearance, ease of physical use and possibly their normal purposes, with less attention paid to specific mechanisms of operation. Most low-prototypicality objects, such as sticks, rulers, or pens, have sufficiently simple and lacking functional mechanisms that these mechanisms are less relevant to object representations. Modeling objects using Lakoff and Johnson's scheme provides principle types of attributes to use when modeling the pretend object substitution process.

\section{Object Substitution Process}

Sawyer's [30] study of communication processes used in pretend play in a preschool classroom provide details on the object substitution process. He found that play communication occurs at one of four play frame levels:

\section{1. in character, in frame \\ 2. in character, blended frame \\ 3. out of character, blended frame \\ 4. out of character, out of frame}

In character, in frame communication (level 1) involves using the voice of an enacted play character and making no reference to the play frame. In character, blended frame communication (level 2) uses a character voice but makes some reference to real-world objects or events not part of the play frame. An example is asking "Jennifer, is this our baby?" where Jennifer is the name of a real-world person while the baby is pretended. Out of character, blended frame communication (level 3) involves directing others 
(that is, not acting as a character) while still referring to objects or events that relate to the play activity. Finally, out of character, out of frame communication (level 4) does not make any reference to the play frame or act as a character within that frame.

Sawyer's aggregated results over all observed communication interactions found children on typically used a framing level of 2 or 3 . This reflects children primarily working in character and mainly referencing pretend objects while still occasionally acting to provide out of character directions to other players or using references to real-world objects and events. We model this level of play reference as the layering of a pretend frame atop an existing physical frame. Agents reason about this mixed frame by blending the real and pretend objects used in object substitution. This draws from the descriptions of pretend play given by the children studied by Sawyers and Carrick and the use of metapragmatic communication found by Sawyer [31,33].

\section{Pretend Object Play Model}

We model pretend object play as the layering of a pretend domain onto a real domain, deriving a blended space for use in communication and reasoning. Agents must map between these domains in both directions. First, when an agent has a desired pretend object it must search the local environment to find a suitable real substitute, and then understand how this substitute can be used as the pretend object. Second, when one agent observes another agent manipulating a real object, it must find an appropriate pretend object to match the given selection of a real object, again basing its interpretation on the pretend context. Our model captures the process of representing and mapping between objects in these domains while respecting the constraints and features specific to both domains.

Real and pretend domains impose differing constraints on pretend object play. The real domain captures knowledge about the workings of real world objects and constraints imposed by those objects on actors for their activities.
These constraints enforce real-world physical limitations such as not being able to pretend a heavy object is substantially lighter than it is, or a rigid object is more flexible than it is. The pretend domain captures the pretend objects of interest. These objects impose constraints on any mapping between the spaces through their distinctive features, such as a pretend sword (typically) being treated as threatening, or used for stabbing. Pretend constraints captures norms of interpretation any player must respect when using a given object in a pretend scenario. Pretending an object is a sword entails assuming others will consider it sharp and used for actions such as stabbing or slashing. Mapping between real and pretend domains involves three primary processes: (1) filtering objects; (2) evaluating object similarity; (3) blending objects.

\section{Pretend To Real Mapping}

During pretend play an agent may have a desired pretend object that it seeks to map onto an available real object. To do so, the agent first filters the set of real objects in its environment. Using a given set of constraining attributes and a threshold for attribute violation does this. Constraining attributes relate to the needs of a particular play context, encoding information such as the need for an object to have perceptual features of a certain kind or for the object to be manipulated in particular ways. Each constraining attribute of each pretend object is checked and the object is rejected if any of its attributes violates these constraints by an amount greater than or equal to the threshold. Computationally, constraints are differences between real and pretend object attribute values. As an example, a pretend sword with a 0.4 DOM value for weight would violate a threshold of 0.2 DOM difference when compared to a real log with a 0.7 DOM value for weight as a constraint. That is, a $\log$ is too heavy to be used as this specific pretend sword. Note that we have fixed the context of pretend play to human-scale domains, making all physical attributes of objects related to the category of human everyday interactions.

\begin{tabular}{|c|c|c|c|c|c|c|c|c|c|}
\hline \multicolumn{9}{|c|}{ Table 1: Objects } \\
\hline & size & length & width & weight & sharpness & grippable & bendability & stabbing & slashing \\
\hline cardboard tube & 0.3 & 0.4 & 0.2 & 0.1 & 0.1 & 0.8 & 0.0 & 0.1 & 0.1 \\
\hline umbrella & 0.3 & 0.4 & 0.2 & 0.2 & 0.1 & 1.0 & 0.3 & 0.2 & 0.1 \\
\hline pen & 0.1 & 0.1 & 0.1 & 0.1 & 0.3 & 1.0 & 0.1 & 0.7 & 0.0 \\
\hline broom & 0.5 & 0.5 & 0.1 & 0.2 & 0.1 & 0.7 & 0.1 & 0.3 & 0.1 \\
\hline & & & & & & & & 0 & 0.0 \\
\hline mace & 0.3 & 0.3 & 0.3 & 0.4 & 0.1 & 0.9 & 0 & 1 \\
\hline dagger & 0.1 & 0.1 & 0.1 & 0.1 & 0.9 & 1.0 & 0 & 0.7 \\
\hline spear & 0.6 & 0.6 & 0.2 & 0.5 & 0.8 & 0.7 & 0.5 & 1.0 & 0.1 \\
\hline sword & 0.4 & 0.4 & 0.2 & 0.4 & 0.9 & 1.0 & 0 & 1.0 & 1.0 \\
\hline broom-sword & 0.5 & 0.5 & 0.1 & 0.2 & 0.1 & 0.7 & 0.1 & 0.3 & 1.0 \\
\hline
\end{tabular}




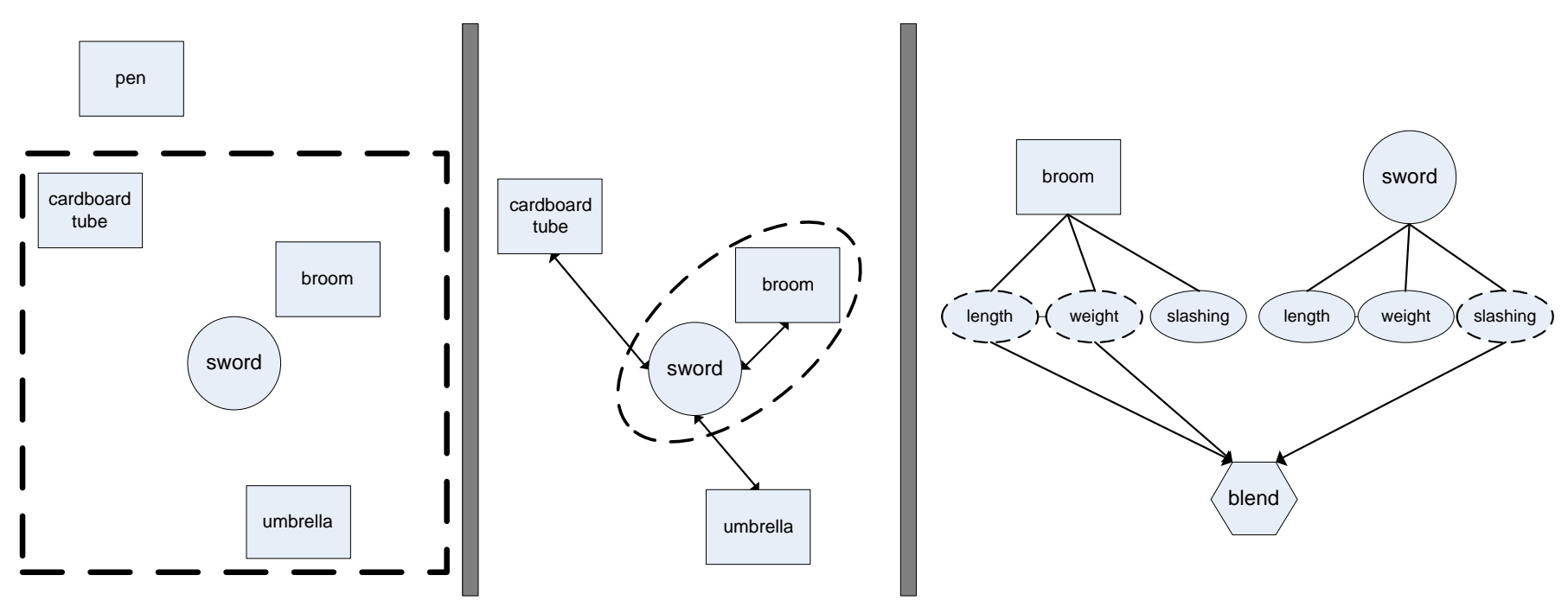

Figure 1: Blending a pretend sword with a real broom. Dashed lines indicate selections made by the agent. Double-headed arrows indicate distance comparisons, single-headed arrows indicate mapping values, and lines indicate attributes of an object. Three steps of the model are shown schematically: filtering objects based on constraints (left), selecting objects based on similarity (center), and blending real objects with iconic values of pretend objects (right).

All remaining pretend objects are next evaluated for their similarity to the given real object. Our computational implementation currently uses a simple Euclidean distance metric using all attributes of both objects. This approach is based on prior work on computational modeling of prototypes [22]. The real object with the least distance to the desired pretend object is selected for use in the object substitution.

Blending occurs by mapping iconic attributes of the pretend object onto the real object. The real object attributes are used by default based on our model of layering the pretend on top of the real domain. Iconic attributes of the pretend object are those attributes that distinguish the pretend object from all other potential pretend objects within its category. Specifically, iconic attributes of an object have values that are the most different from all other objects in Third, the agent blends the real and pretend objects for the purposes of reasoning. As before, the real object is used as a baseline to capture the process of layering the pretend domain upon the real. Iconic values of the pretend object are mapped onto the blended object and used as the final result.

the set according to that attribute. Our algorithm calculates iconicity as the sum of Euclidean distances of a pretend object's attribute DOM from the DOM of all other pretend objects for that attribute. Iconicity values are normalized within categories of objects and an attribute is considered iconic for an object if it is within a given range from the maximum value. The blending process finds all iconic values of the pretend object and maps these onto the blended object, replacing the physical object attribute values. This allows the final blended object to retain the constraints of its real form while allowing an agent to reason about the pretend features of the object.
Observing the use of a real object in a play context, an agent must find the potential pretend object being represented and understand how it relates to the current play activity. This process also occurs when an agent observes some real object in the environment and seeks to give it a pretend instantiation.

First, the agent filters out all pretend objects based on a set of constraints and a threshold for constraint violation. As above constraints reflect the physical limitations of the domain and are used to reduce the space of potential pretend object candidates.

Second, the agent calculates the similarity of the real object to all remaining pretend objects. This process uses the same Euclidean distance minimization process as above and results in a maximally similar real object.

\section{Example: Pretend Sword}

As an example, consider an agent attempting to map an imaginary sword to a real object among an available cardboard tube, umbrella, pen, and broom (Figure 1 and Table 1). First, the agent focuses on the constraining attributes of overall size, length, width, and thickness of the real objects, using a threshold of an absolute difference of 0.2 (Figure 1, left). The agent rejects the pen for being too short and too small (both differences of 0.3 ) to be used as a pretend sword.

Second, the agent evaluates the similarity between each remaining real object and the pretend sword (Figure 1, center). Distance to objects is calculated as the sum of the Euclidean distances of all attributes. The normalized inverse of this quantity determines similarity. According to this measurement the broom is most similar to a sword (1.0 similarity), followed by the umbrella (0.55) and then cardboard tube (0). 
Third, the agent evaluates each attribute of the sword to determine its iconic attributes. Each pretend object is compared to all other pretend objects along a particular attribute. The iconicity value for that attribute for that object is calculated as the sum of the Euclidean distances of that objects attribute value from all other objects. For example, the sword's iconicity for its slashing attribute is calculated as:

$$
(1.0-0.0)^{2}+(1.0-0.7)^{2}+(1.0-0.1)^{2}=1.9
$$

After normalizing iconicity values across all objects this becomes a value of 1.0. The sword is iconic for being most typically used for slashing among the pretend objects. Among all attributes this is the only iconic value of the sword.

Finally, the agent produces a blended object that integrates both the real broom with the pretend sword (Figure 1, right). The blended sword matches the real broom on all attributes except the sword's iconic slashing attribute. The result is a broom-sword blend that functions for slashing.

\section{Discussion and Future Work}

We have presented a synthesis of research on pretend play in order to identify the key features necessary for implementing computational play agents. Pretend play involves navigating dual real and pretend domains while working on a blended level that takes elements from both domains. Pretend domains include information about themes, scripts, and rules to guide activities based on everyday experiences and the surrounding media environment. Navigation between the pretend and real domains involves constructing a blended frame using analogical and conceptual blending processes. This frame is continually manipulated using metacommunicative and metapragmatic processes that balance coherence with existing indexical presuppositions and open-ended change using indexical entailments. Pretend object play grounds representation and process features of this model in graded category representations and analogical and blending processes.

Our model of pretend object play requires human evaluation. The metrics for similarity and iconicity both are designed to be relatively insensitive to the exact details of the attribute data they are given as they serve only to impose an ordering on objects. Evaluation of the model will require gathering human data for attribute values and specific objects used in play categories. As estimations of similarity are context-dependent this data collection will need to be grounded in a particular play domain and particular real-world setting. Additionally, we also need to collect human data on the constraints of relevance to physical mappings.

Further specifying the pretend play model as a whole requires greater specification of the processes involved in selecting and using play contexts and interacting between pretending agents. We have assumed a given pretend context for our pretend object play model. During pretend play many contexts are found and integrated in real time. How do agents select a pretend domain, such as a fairy tale world, to use in pretend play? What content from these domains do players use and how do they select it? When using a particular play frame, how do players draw multiple objects or stories from them and integrate them? Research on play has already found narratives, pretend worlds, and digital game rules are appropriated, but has left unspecified how this process occurs. As many pretend domains are integrated in most pretend play sessions, how are these additional domains selected and combined? Conceptual blending and analogy are likely involved in the appropriation process, but the details of their guiding constraints and considerations remain unclear.

Future research also needs to specify the constraints of particular real world play contexts. Play behavior differs between playgrounds and indoors, as well as when playing with siblings, friends or relative strangers. Incorporating play agents into real-world applications will require further investigation of the social and cultural constraints that frame pretend play in these domains. Additional extensions of this work must model the metapragmatic processes of recognizing and incorporating such frames into activities during the course of the activities themselves.

Pretend play interactions use metapragmatics to negotiate the ongoing play activity. What content is conveyed using these statements? Indexical presuppositions and entailments provide an initial framework for how agents communicate about these appropriations. However, Sawyer notes the need to understand the relation between these statement types and the content they convey [31]. What is the nature of the indexical presuppositions and indexical entailments that players use in terms of their content and selection? How do players make decisions about the balance between existing structure and open-ended freedom? Such processes are central to enabling coherent play experiences and will be requisite for any agent that interacts with human players if it is to keep them engaged in playful activity and drive players to reap the benefits of play.

\section{REFERENCES}

1. Bartneck, C. and Forlizzi, J. A design-centred framework for social human-robot interaction. Robot and Human Interactive Communication, 2004. ROMAN 2004. 13th IEEE International Workshop on, (2004), 591-594.

2. Bateson, G. A Theory of Play and Fantasy. In Steps to an ecology of mind. Chandler Pub. Co., San Francisco, CA, 1972, 177-193.

3. Boden, M.A. Computer models of creativity. AI Magazine 30, 2009, 23-35.

4. Breazeal, C. and Scassellati, B. Infant-like social interactions between a robot and a human caregiver. Adaptive Behavior 8, 1 (2000), 49. 
5. Breazeal, C. Toward sociable robots. Robotics and Autonomous Systems 42, 3-4 (2003), 167-175.

6. Breazeal, C. Social interactions in HRI: The robot view. Systems, Man, and Cybernetics, Part C: Applications and Reviews, IEEE Transactions on 34, 2 (2004), 181186.

7. Brown, S.L. and Vaughan, C.C. Play: How it shapes the brain, opens the imagination, and invigorates the soul. Avery Pub Group, 2009.

8. Caillois, R. Man, play, and games. University of Illinois Press, Urbana, 2001.

9. Falkenhainer, B. and Dedre, K.D.F. The structuremapping engine: Algorithm and examples. Artificial intelligence 41, 1 (1989), 1-63.

10. Fauconnier, G. and Turner, M. Conceptual integration networks. Cognitive Science: A Multidisciplinary Journal 22, 2 (1998), 133-187.

11. Fauconnier, G. and Turner, M. The way we think: Conceptual blending and the mind's hidden complexities. Basic Books, 2003.

12. Forbus, K.D., Gentner, D., and Law, K. MAC/FAC: A model of similarity-based retrieval. Cognitive Science 19, 2 (1995), 141-205.

13. Fromberg, D.P. A review of research on play. In The early childhood curriculum: Current findings in theory and practice. 1999, 27-53.

14. Fuller, D. and Magerko, B. Shared mental models in improvisational performance. Proceedings of the Intelligent Narrative Technologies III Workshop, (2010), 1-6.

15. Gaster, S. Urban Children's Access to their Neighborhood. Environment and Behavior 23, 1 (1991), 70.

16. Horst, P.G.L., Mahendran, D., Martinez, K., et al. Hanging Out, Messing Around, Geeking Out: Living and Learning with New Media. Cambridge: MIT Press, Forthcoming.

17. Huizinga, J. Homo Ludens: A Study of the Play-Element in Culture. Taylor \& Francis, 2003.

18. Jenkins, H. Complete freedom of movement: Video games as gendered play spaces. From barbie to mortal kombat: Gender and computer games, (1998).

19. Kosko, B. Fuzzy thinking: The new science of fuzzy logic. 1993.

20. Lakoff, G. and Johnson, M. Metaphors we live by: With a new afterword. Chicago: The University of Chicago Press, 2003.

21. Lakoff, G. Women, fire, and dangerous things: What categories reveal about the mind. University of Chicago press, 1987.

22. Lesot, M.J. Similarity, typicality and fuzzy prototypes for numerical data. 6th European Congress on Systems Science, Workshop on Similarity and resemblance, (2005).

23. Lillard, A. and Else-Quest, N. The Early Years: Evaluating Montessori. Science 313, 5795 (2006), 1893-1894.
24. Magerko, B., Fiesler, C., and Baumer, A. Fuzzy MicroAgents for Interactive Narrative. Proceedings of the Sixth Annual AI and Interactive Digital Entertainment Conference, (2010).

25. Morgenthaler, S.K. The meanings in play with objects. In Play from birth to twelve and beyond: contexts, perspectives, and meanings. 1998, 359-367.

26. Nourot, P.M. Sociodramatic play: Pretending together. In Play from birth to twelve and beyond: contexts, perspectives, and meanings. 1998, 378-391.

27. Power, T.G. Play and exploration in children and animals. 2000.

28. Proyer, R.T. Being playful and smart? The relations of adult playfulness with psychometric and self-estimated intelligence and academic performance. Learning and Individual Differences, (2011).

29. Rosch, E. Principles of categorization. In Concepts: core readings. 1999, 189-206.

30. Sawyer, R.K. Levels of Analysis in Pretend Play

Discourse: Metacommunication in Conversational Routines. Play and educational theory and practice, (2003).

31. Sawyer, R.K. Pretend play as improvisation : conversation in the preschool classroom. L. Erlbaum Associates, Mahwah N.J., 1997.

32. Sawyer, R.K. Improvisation and Narrative. Narrative Inquiry 12, 2 (2002), 319-349.

33. Sawyers, J.K. and Carrick, N. Symbolic play through the eyes and words of children. Play and educational theory and practice, (2003), 159.

34. Scheeff, M., Pinto, J., Rahardja, K., Snibbe, S., and Tow, R. Experiences with Sparky, a social robot. Socially Intelligent Agents, (2002), 173-180.

35. Sutton-Smith, B. The role of toys in the instigation of playful creativity. Creativity Research Journal 5, 1 (1992), 3-11.

36. Sutton-Smith, B. The ambiguity of play. Harvard University Press, Cambridge Mass., 2001.

37. Veale, T. and Donoghue, D.O. Computation and blending. Cognitive Linguistics 11, 3/4 (2000), 253282.

38. Zhu, J. Story and Text Generation through Computational Analogy in the Riu System. (2010). 